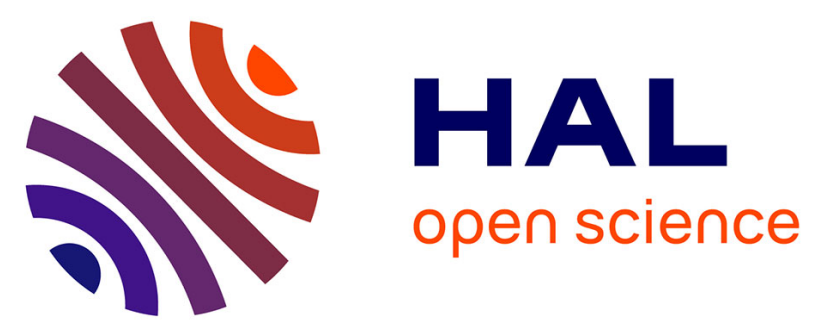

\title{
A Fixed Mesh Numerical Method for Modelling the Flow in Liquid Composites Moulding Processes Using a Volume of Fluid Technique
}

\author{
Juan Antonio Garcia, Llanos Gascón, Francisco Chinesta
}

\section{- To cite this version:}

Juan Antonio Garcia, Llanos Gascón, Francisco Chinesta. A Fixed Mesh Numerical Method for Modelling the Flow in Liquid Composites Moulding Processes Using a Volume of Fluid Technique. Computer Methods in Applied Mechanics and Engineering, 2003, 192, pp.877-893. 10.1016/S00457825(02)00604-7 . hal-00020741

HAL Id: hal-00020741

https://hal.science/hal-00020741

Submitted on 17 Apr 2018

HAL is a multi-disciplinary open access archive for the deposit and dissemination of scientific research documents, whether they are published or not. The documents may come from teaching and research institutions in France or abroad, or from public or private research centers.
L'archive ouverte pluridisciplinaire HAL, est destinée au dépôt et à la diffusion de documents scientifiques de niveau recherche, publiés ou non, émanant des établissements d'enseignement et de recherche français ou étrangers, des laboratoires publics ou privés. 


\title{
A fixed mesh numerical method for modelling the flow in liquid composites moulding processes using a volume of fluid technique
}

\author{
J.A. García ${ }^{\mathrm{a}}$, Ll. Gascón ${ }^{\mathrm{b}, *}$, F. Chinesta ${ }^{\mathrm{c}}$ \\ a Depto. Ing. Mecánica y Materiales, Universidad Politécnica de Valencia, Camino Vera s/n, 46022 Valencia, Spain \\ ${ }^{\mathrm{b}}$ Depto. Matemática Aplicada, Universidad Politécnica de Valencia, Camino Vera s/n, 46022 Valencia, Spain \\ ${ }^{\mathrm{c}}$ Laboratoire de Mécanique des Systèmes et des Procédés, UMR CNRS-ENSAM-ESEM, 151 Boulevard de l'Hôptital, \\ F-75013 Paris, France
}

This paper presents the development and application of a numerical procedure for the simulation of the flow phenomena involved in composite manufacturing processes. The kinematic resolution combines a discretization of the volume fraction evolution equation and the verification of the movement and the incompressibility equations in a fixed mesh. We consider a variational formulation of Darcy's flow weighted by the volume fraction and the variational formulation of a pseudo-behaviour associated with the empty part of the domain weighted by its complementary function. For the integration of the advection equation, which governs the volume fraction evolution, a second-order scheme with flux limiters has been used. In order to validate the proposed method, numerical results in one and two dimensions are presented.

Keywords: Composite forming processes; Liquid injection moulding; Advection equation; Fixed mesh simulation; Flux limiters

\section{Introduction}

Liquid composites moulding (LCM) processes and, particularly, resin transfer moulding (RTM) are being increasingly used in the manufacture of fiber-reinforced composite materials. Many of the final qualities of the piece are determined during the injection and the curing. Therefore, the numerical simulation of these processes can help us to optimise the design and manufacture of these pieces. A good composite product can be only obtained when the reinforcement is fully impregnated by the resin and

\footnotetext{
${ }^{*}$ Corresponding author.

E-mail addresses: jugarcia@mcm.upv.es (J.A. García), llgascon@mat.upv.es (L. Gascón), francisco.chinesta@paris.ensam.fr (F. Chinesta).
} 
properly cured. Several numerical methods have been proposed to simulate the resin flow in RTM processes.

Once the governing equations in the macroscopic scale are established, one needs to introduce numerical techniques for their integration. The numerical treatment involves the description of a moving free surface (the flow front), a fundamental issue in the simulation of mold filling processes. The shape and location of this flow front can be computed, and then the boundary of the saturated domain must be continuously redefined at each time step during the integration. Two different kind of numerical procedures can be used to simulate injection processes: moving grid techniques [11] and fixed grid approaches $[1,18]$. The moving grid techniques are based on frequent remeshing of the resin saturated part of the mold. The accuracy of the moving grid techniques is generally good, but new meshes must be created, which results high CPU time consuming. Methods have been developed to reduce the frequency of remeshing about one decade ago (see the works of Tezduyar [13-15] and its review paper [16] and the references therein). However, in some classes of problems it may not be possible to reduce the remeshing frequency to a practical level. Moreover, an adequate management of the flow front in the neighbouring of the mold walls and the interaction between two fronts (molds with inserts or multiple injection ports) must be incorporated in the numerical scheme.

The numerical method used in this paper is based on the resolution of the equation governing the resin presence function (resin volume fraction) in a fixed mesh. Due to the problems associated with the mass conservation, it is very frequent to use a mesh for the computation of the pressures field and other one to update the flow domain. This method needs two discretisations of the mold, one to solve Darcy's equation and another to actualise the fluid domain. There is a control volume cell associated with each node of the finite elements mesh. The main advantage of the control volume method is that it satisfies automatically the mass conservation. The flow front location is defined by the partially filled control volumes. Once the pressure field is computed at a given time, the velocity in each element is obtained from the Darcy's equation. Then the flow across each control volume can be easily calculated, and the volume fraction updated. The flow governing equations are applied only in the filled region and a null pressure is imposed at the nodes whose control volumes are partially filled or empty. At each iteration the time step selected is chosen as the shortest required to fill completely a control volume. On the other hand, Advani [1] use a modified element technique in which the elements and the control volumes coincide, it eliminates the error due to the deform elements and guarantees the mass conservation.

In the method proposed by Trochu et al. [18], the control volumes coincide with the finite elements. In this case, however, the pressure field cannot be obtained from the usual continuous interpolation between elements, because in that case, the velocity becomes discontinuous between elements and the conservation of the resin mass is not satisfied. Hence, Trochu proposes the use of a non-conforming linear finite element approximation of the pressure field, in which, in spite of that the pressure is discontinuous on the element boundaries (except at the middle point), the velocity remains continuous between elements, yielding a numerical model that satisfies locally the resin mass conservation.

The numerical model proposed in this work uses the same mesh in the pressure calculation and in the fluid domain updating. However, it employees a linear and continuous interpolation for the pressure field (conforming method). This strategy is based on the discretisation of the flow variational formulation in the domain occupied by fluid and the variational formulation of a null pressure condition in the empty part of the mold. A weighting formulation will be considered in the partially filled elements, where the weight function depends on the fluid fraction in each element. The fluid fraction will be updated by means of a second-order upwinding technique using flux limiters, due to the hyperbolic character of the equation governing its evolution (advection equation).

The numerical resolution of hyperbolic equations has been extensively treated during the last decades due to the difficulty of obtaining schemes that reproduce with great accuracy the solution in smooth regions as well as in presence of discontinuities (see, for instance $[8,10,17]$ ). First-order upwind schemes have been 
used to approximate solutions of conservation laws. These methods have a strong numerical dissipation in the neighbourhood of discontinuities giving a low accuracy in smooth regions of the solution. In order to avoid this problem, some new shock-capturing finite difference schemes have been proposed. These techniques are of second-order in smooth regions of the solution, without generating the spurious oscillations associated with the conventional second-order schemes, such as Lax-Wendroff, in the presence of discontinuities. An important kind of these schemes was introduced by Harten [7]. They are the total variation diminishing (TVD) schemes, which guarantee that monotone functions remain monotone and second-order accuracy in the numerical solution. One version of these techniques are the flux limiters, introduced by Sweby [12], and based on the definition of hybrid schemes that use second-order fluxes in smooth regions of the solution, with a limitation of the numerical fluxes, to avoid numerical oscillations, in the vicinity of the discontinuities.

The main aim of this work is the description of a numerical model for RTM simulations in which the fluid domain is updated by using a second-order scheme with flux limiters. We observe, in the onedimensional case, that when the injection takes place through one of the domain boundaries, the original version of the flux limiters gives excellent results. However, this technique is not adapted to multi-point injection. For this reason, a new version of the flux limiters will be proposed that provide excellent results regardless of the position of the injection points. This formulation will be adapted to treat also 2D models. In order to select the optimal numerical scheme, different flux limiters (Minmod, Superbee and Monotonized Centered) have been adapted, tested and compared, to update the volume fraction in the simulation of the RTM process. Finally, this scheme has been incorporated to the new aforementioned technique developed by the authors for RTM simulation.

The organization of this paper is as follows. In Section 2, we present the RTM flow model, whose numerical discretisation will be described in Section 3. In order to compute the pressure and velocity fields we use an extended variational formulation that combines the governing equations associated with the filled and empty domains. Then a new version of the flux limiters technique has been developed in one- and twodimensional cases in order to update the volume fraction in the context of the RTM process. Section 4 is devoted to present some numerical results, to finish with the concluding remarks given in Section 5 .

\section{Governing equations}

The resin impregnation is usually modelled as a flow in porous media. The model equations which describe this process are given by:

- Darcy's law, which states that the flow velocity, $\underline{v}$, is proportional to the pressure gradient, according to

$$
\underline{v}=-\frac{\underline{K}}{\underline{\mu}} \nabla p,
$$

where $\underline{\underline{K}}$ is the preform permeability tensor, $\mu$ is the viscosity of the fluid and $p$ is the pressure.

- The incompressibility of the fluid, expressed as

$$
\operatorname{Div} \underline{v}=0,
$$

- The evolution of the resin volume fraction I (fluid presence function), which is governed by the following transport equation:

$$
\frac{\partial I}{\partial t}+\underline{v} \cdot \nabla I=0
$$




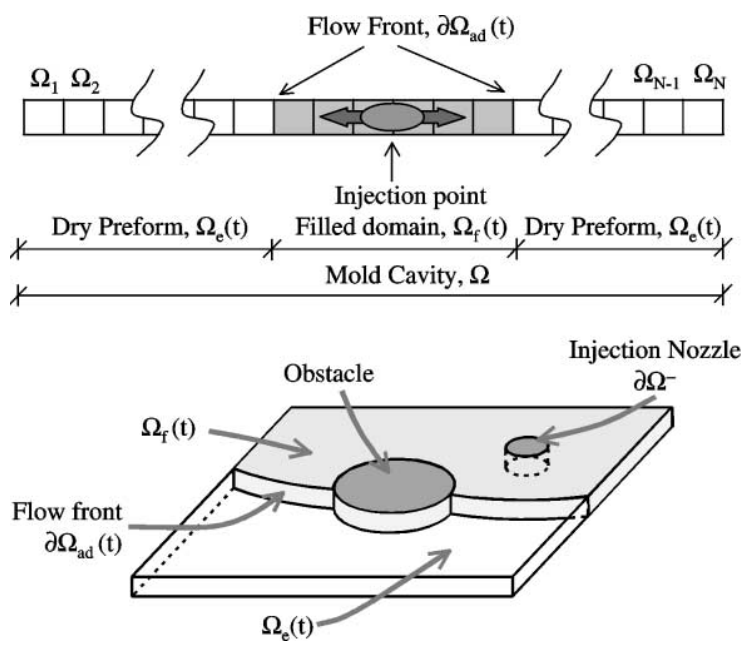

Fig. 1. One and two-dimensional models.

The value of the variable $I$ measures the degree of saturation and will be used to know the position of the flow front at each time step. The volume fraction, $I$, is defined as

$$
I(\underline{x}, t)= \begin{cases}1 & \text { if } \underline{x} \in \Omega_{\mathrm{f}}(t), \\ 0 & \text { if } \underline{x} \notin \Omega_{\mathrm{f}}(t),\end{cases}
$$

where $\Omega_{\mathrm{f}}(t)$ denotes the fluid domain at time $t$ (see Fig. 1).

If Eq. (1) is introduced into Eq. (2), the pressure at each point of the domain occupied by the fluid results from the solution of

$$
\operatorname{Div}\left(\frac{\underline{\underline{K}}}{\mu} \nabla p\right)=0 \text {. }
$$

If we assume that the permeability and the viscosity are constants and that the permeability is orthotropic, then the permeability tensor can be written in a diagonal form in the principal directions and Eq. (4) can be simplified

$$
\left(\frac{k_{x x}}{\mu} \frac{\partial^{2} p}{\partial x^{2}}+\frac{k_{y y}}{\mu} \frac{\partial^{2} p}{\partial y^{2}}\right)=0
$$

where $k_{x x}$ and $k_{y y}$ are the preform permeability in the principal directions.

The whole domain (mold) is denoted by $\Omega$, being $\partial \Omega$ its boundary

$$
\Omega=\Omega_{\mathrm{f}}(t) \cup \Omega_{\mathrm{e}}(t),
$$

where $\Omega_{\mathrm{f}}(t)$ denotes the resin saturated part of the mold at time $t$, and $\Omega_{\mathrm{e}}(t)$ is the empty part of the domain. The boundaries of $\Omega_{\mathrm{f}}(t)$ and $\Omega_{\mathrm{e}}(t)$ are $\partial \Omega_{\mathrm{f}}(t)$ and $\partial \Omega_{\mathrm{e}}(t)$, respectively. The filled and empty domains of the mold has a common boundary, which corresponds to the fluid flow front $\partial \Omega_{a d}(t)$ (see Fig. 1).

The boundary conditions are:

- The pressure gradient in the normal direction to the mold walls is zero. Physically this means that material cannot leaves the mold cavity through the mold walls.

- The pressure or the flow rate are prescribed on the inflow boundary (injection nozzle), $\partial \Omega^{-}$, i.e. 


$$
\begin{aligned}
& p\left(x \in \partial \Omega^{-}\right)=P_{i} \quad \text { or } \quad \underline{v}\left(\underline{x} \in \partial \Omega^{-}\right)=\underline{v}_{i}, \\
& \partial \Omega^{-}=\{\underline{x} \in \partial \Omega, \underline{v}(\underline{x}) \cdot \underline{n}(\underline{x})<0\},
\end{aligned}
$$

where $\underline{n}(\underline{x})$ is the unit outwards vector, defined on the boundary at point $\underline{x}$.

- The pressure is zero at the flow front, that is

$$
p\left(\underline{x} \in \partial \Omega_{a d}(t)\right)=0 .
$$

Moreover, we assume that at time $t=0$, the mold is empty, i.e.

$$
I(\underline{x}, t=0)= \begin{cases}0 & \text { if } \underline{x} \in \Omega, \\ 1 & \text { if } \underline{x} \in \partial \Omega^{-} .\end{cases}
$$

\section{Numerical modelling}

In this section we propose an efficient technique to simulate the flow in a porous medium. The resolution of the governing equations will be performed by means of a conforming finite element Galerkin technique, whereas that for the fluid domain updating a volume of fluid (VOF) technique [9] will be employed. The resolution algorithm is based in solving until the complete filling of the mold (while $\left|\Omega_{\mathrm{f}}(t)\right|<|\Omega|$ ), the next three problems:

Step 1: Obtain the pressure field using a finite elements discretisation of the variational formulation extended to the whole domain.

Step 2: Compute the velocity field from the Darcy's law.

Step 3: Update the fluid presence function I (which defines the fluid and empty domains) by integrating the transport equation governing its evolution.

\subsection{Flow kinematics resolution}

In order to achieve steps 1 and 2, we propose to combine the filled domain and the empty domain behaviours in the same variational formulation. We note that the governing equation to impose in the filled domain is given by Eq. (4) whereas in the empty region of the mold a null pressure will be imposed

$$
p=0 \text { in } \Omega_{\mathrm{e}}(t) .
$$

Thus, the variational formulation associated with the empty region is given by

$$
\int_{\Omega_{\mathrm{e}}(t)} p p^{*} \mathrm{~d} \Omega=0
$$

where $p^{*}$ denotes the weighting function, whereas the variational formulation of Eq. (4) in the fluid domain results

$$
\int_{\Omega_{\mathrm{f}}(t)} \nabla p^{*} \cdot \frac{\underline{\underline{K}}}{\mu} \nabla p \mathrm{~d} \Omega=0
$$

Both variational formulations (Eqs. (6) and (7)) can be combined and extended to the whole domain

$$
\int_{\Omega}\left(I \nabla p^{*} \cdot \frac{\underline{\underline{K}}}{\mu} \nabla p+\alpha(I)(1-I) p p^{*}\right) \mathrm{d} \Omega=0
$$


where $\alpha(I)$ is required in order to guarantee the dimensional homogeneity. In a former work [4], the authors have analyzed the effect and optimal choice of this function.

Since in the practice, we cannot avoid the existence of partially filled elements, with $0<I<1, I$ could be replaced by a general weighting function $f(I)$

$$
\int_{\Omega}\left(f(I) \nabla p^{*} \cdot \frac{\underline{\underline{K}}}{\mu} \nabla p+\alpha(I)(1-f(I)) p p^{*}\right) \mathrm{d} \Omega=0
$$

We observe that Eq. (8) consists in two terms, one that models the flow in the domain occupied by fluid and the other one that models the pseudo-behaviour (null pressure) imposed in the empty domain. In the flow front neighbouring, where $0<I<1$, a weighting of both behaviours is applied. In the case of taking the weighting function equal to the volume fraction, $f(I)=I$, the previous expression constitutes a linear weighting of the flow variational formulation, valid in the domain occupied by the fluid, where $I=1$, and the null pressure condition to be imposed in the empty domain, where $I=0$. In any case, the weighting function, $f(I)$, must vanish in the empty domain and must take a unit value in the filled domain. If we want to avoid a weighting of both behaviours in the partially filled elements, a discontinuous function $f(I)$ can be defined as it is shown in Fig. 2, by using a jump function, where $I_{\text {th }}$ represents at threshold value, from which we considered an element as full filled $\left(I>I_{\text {th }}\right)$ or empty $\left(I<I_{\text {th }}\right)$ in the kinematics computation.

The criterion for an optimal choice of $\alpha(I)$ and the weighting function $f(I)$ is that the resulting scheme should be conservative and have a minimal numerical diffusion in the flow front location. A detailed study about the choice for these functions can be found in [4,5].

The discretisation of the extended variational formulation is carried out by using a standard finite elements method. We assume the domain $\Omega$ divided in $N$ elements

$$
\Omega=\bigcup_{e=1}^{N} \Omega^{e}
$$

then the FE discretisation of Eq. (8) becomes

$$
\sum_{e=1}^{N}\left\{\int_{\Omega^{e}}\left[\nabla p^{*} \cdot \frac{\underline{\underline{K}}}{\mu} \nabla p^{e} f\left(I^{e}\right)+\left(1-f\left(I^{e}\right)\right) \alpha\left(I^{e}\right) p^{e} p^{*}\right] \mathrm{d} \Omega\right\}=0
$$

from which results the pressure distribution at each time step (Step 1). Then, the velocity is computed from Darcy's law (1) (Step 2) and the flow front and fluid domain updating will be achieved by solving Eq. (3) with the just computed kinematics (Step 3) whose numerical treatment is described in the next section.

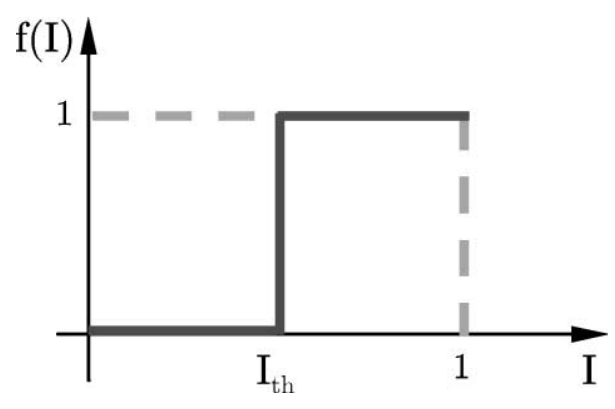

Fig. 2. Definition of $f(I)$ as a jump function. 
Remark 1. In spite of that the discretisation of the variational formulation extended to the whole domain given by Eq. (8) involves the unknown nodal pressure at each node of the whole domain, in fact, in the resolution, a null pressure is imposed in the nodes surrounded by fluid-free elements, in order to improve the computational efficiency.

\subsection{Fluid domain evolution and flow front tracking}

Once the pressure and velocity fields have been calculated at time $t$, the fluid fraction can be updated in order to compute the fluid domain and determine the new position of the flow front (Step 3). The evolution of the variable $I$ is given by the aforementioned linear advection equation

$$
\frac{\partial I}{\partial t}+\underline{v} \cdot \nabla I=0
$$

with the initial condition

$$
I(\underline{x}, t=0)= \begin{cases}0 & \text { if } \underline{x} \in \Omega \\ 1 & \text { if } \underline{x} \in \partial \Omega^{-}\end{cases}
$$

and the boundary condition $I\left(\underline{x} \in \partial \Omega^{-}, t\right)=1$.

Eqs. (9) and (10) define an initial value problem for an hyperbolic conservation law. There are several and sophisticated schemes for the numerical resolution of these problems (see, for instance, [6-8,10,17]), most of them are characterized by checking the TVD property [7] that allows to define second-order nonoscillatory schemes. These schemes can be classified in two main groups: slope limiters $[19,20]$ and flux limiters [2,3,12]. Our objective is apply the flux limiters suggested by Sweby in [12] to RTM processes and compare the influence of different flux limiters found in the literature in the numerical solution accuracy.

Remark 2. The proposed strategy can be considered as a particular case of the EDICT strategy proposed by Tezduyar (see [16] and the references therein) when an explicit formulation is used and different stabilization techniques are applied in the discretisation of the advection equation governing the fluid domain evolution. In our case, no multi-level meshes are employed.

\subsubsection{A flux limiter strategy for one-dimensional problems}

Firstly we describe the original version of that technique and later we will propose some improvements in order to obtain more accurate schemes to simulate general injection processes.

In order to integrate the transport equation, we take into account the expression of a conservative scheme defined by

$$
I_{i}^{n+1}=I_{i}^{n}-\lambda\left(\hat{f}_{i+1 / 2}-\hat{f}_{i-1 / 2}\right),
$$

where the subscript denotes the considered element, the superscript indicates the time step, and $\lambda$ the ratio between the time step and the element size, i.e. $\lambda=\Delta t / \Delta x$, which is assumed constant. From now on, and for a sake of simplicity, we will omit the superscript in the variables corresponding to the time step $n$ and we define $f_{k}=I_{k} v_{k}$, where $v_{k}$ is the velocity in the element $k$.

The particular case in which the average flux is obtained from a simple Taylor's development in the intermediate time, i.e., the scheme given by Eq. (11) with $\hat{f}_{i+1 / 2}$ defined by

$$
\hat{f}_{i+1 / 2}^{\mathrm{LW}}=f_{i+1 / 2}^{n+1 / 2}=\frac{1}{2}\left\{f_{i}+f_{i+1}-\left(\lambda v_{i+1 / 2}\right)\left(f_{i+1}-f_{i}\right)\right\},
$$

describes the well-known second-order scheme of Lax-Wendroff, that generates numerical oscillations in the regions involving solution discontinuities. In the previous expression, 


$$
v_{i+1 / 2}=\left(v_{i}+v_{i+1}\right) / 2 .
$$

On the other hand, the first-order upwind scheme is obtained substituting in Eq. (11) the average flux $\hat{f}_{i+1 / 2}$ by

$$
\hat{f}_{i+1 / 2}^{\mathrm{UP}}=\frac{1}{2}\left\{f_{i}+f_{i+1}-\operatorname{sign}\left(\lambda v_{i+1 / 2}\right)\left(f_{i+1}-f_{i}\right)\right\} .
$$

Thus, the second-order flux average defining the Lax-Wendroff scheme can be written as the sum of the first-order upwind flux with an additional second-order antidiffusive term that provide the second-order accuracy, i.e.

$$
\hat{f}_{i+1 / 2}^{\mathrm{LW}}=\hat{f}_{i+1 / 2}^{\mathrm{UP}}+\frac{1}{2}\left[\operatorname{sign}\left(\lambda v_{i+1 / 2}\right)-\left(\lambda v_{i+1 / 2}\right)\right]\left(f_{i+1}-f_{i}\right) .
$$

The Sweby's limiters [12] are based on limiting this last term (antidiffusive flux), so that it is cancelled in the discontinuity regions, preserving the TVD property and, therefore, a non-oscillatory behaviour. In this way, the discretisation corresponding to the Sweby's limiters is carried out considering in Eq. (11) the next definition for the flux

$$
\hat{f}_{i+1 / 2}^{\mathrm{SW}}=\hat{f}_{i+1 / 2}^{\mathrm{UP}}+\frac{1}{2} \chi\left(r_{i+1 / 2}\right)\left[\operatorname{sign}\left(\lambda v_{i+1 / 2}\right)-\left(\lambda v_{i+1 / 2}\right)\right]\left(f_{i+1}-f_{i}\right),
$$

where $\chi$ is the limiting function that avoids the presence of oscillations and the parameter $r_{i+1 / 2}$ depends on two consecutive gradients of the solution

$$
r_{i+1 / 2}= \begin{cases}\frac{I_{i}-I_{i-1}}{I_{i+1}-I_{i}} & \text { if } v_{i+1 / 2} \geqslant 0, \\ \frac{I_{i+2}-I_{i+1}}{I_{i+1}-I_{i}} & \text { if } v_{i+1 / 2}<0 .\end{cases}
$$

In general, $\chi(r)=0$ if $r \leqslant 0$ to guarantee a first-order accuracy in the neighbourhood of a discontinuity, since $r \leqslant 0$ implies that the slopes of the solution have opposite signs, i.e. that $I_{i}$ is an extreme of the solution (see Fig. 3). On the other hand, it is necessary that $\chi(1)=1$ to obtain a second-order scheme in the zones where the solution is smooth enough. The limiter function should be chosen verifying both conditions and maximizing the antidiffusive flux. In general, the choice $\chi=0$ gives rise to the original first-order upwind flux, whereas the choice $\chi=1$ defines the second-order Lax-Wendroff numerical flux.

Different flux limiters can be found in the literature [10,12]. In this paper, we analyze the following classic limiters:

$$
\begin{array}{ll}
\chi_{\mathrm{MM}}(r)=\max \{0, \min (r, 1)\} & \text { Minmod, } \\
\chi_{\mathrm{SB}}(r)=\max \{0, \min (2 r, 1), \min (r, 2)\} & \text { Superbee, } \\
\chi_{\mathrm{MC}}(r)=\max \{0, \min (2 r, 2,(1+r) / 2)\} & \text { Monotonized Centered. }
\end{array}
$$

We have observed that in the RTM simulation these schemes cannot be applied in the case of injecting through an internal point of the mold or when two or more injection points are used. This behaviour is basically due to the fact that the first-order upwind scheme and the flux limiters are based on the sign of the average velocity between two consecutive elements, which can be different to the sign of the velocity of the

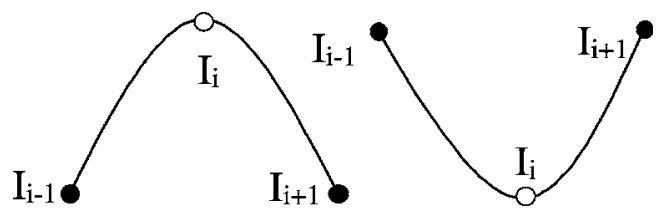

Fig. 3. Localization of local extremes. 

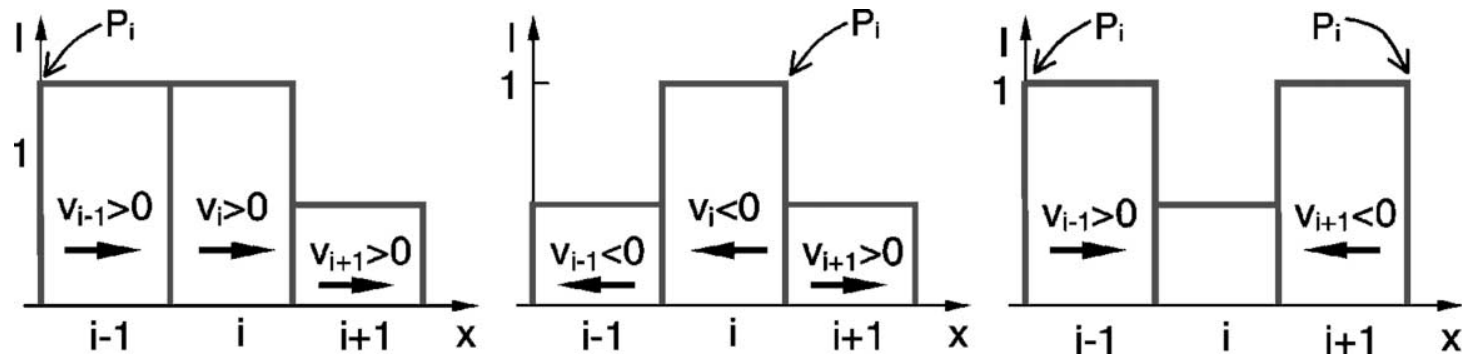

Fig. 4. One-dimensional flow: different situations.

adjacent elements. In order to illustrate this fact we illustrate in Fig. 4 three different situations according to the location of the injection point(s).

We can notice in Fig. 4 that to identify if the flow is coming or leaving the element $i$ it seems reasonable to check the sign of the velocity of adjacent elements and not the sign of the average velocity between elements. When the injection point is located in an extreme of the mold the aforementioned schemes describe correctly this process because

$$
\operatorname{sign}\left(v_{i+1 / 2}\right)=\operatorname{sign}\left(v_{i}\right), \quad \forall i
$$

However, in case of injecting through an internal point or using several points, this equality is not verified always and the previous schemes cannot be applied.

In order to define accurate and general numerical schemes, we propose the following improvements: firstly, we define the first-order upwind scheme

$$
I_{i}^{n+1}=I_{i}^{n}-\lambda\left(f_{i+1 / 2}^{+}-f_{i-1 / 2}^{-}\right),
$$

where

$$
\begin{aligned}
& f_{i+1 / 2}^{+}=\frac{1}{2}\left\{f_{i}+f_{i+1}-\operatorname{sign}\left(v_{i+1}\right)\left(f_{i+1}-f_{i}\right)\right\}, \\
& f_{i+1 / 2}^{-}=\frac{1}{2}\left\{f_{i}+f_{i+1}-\operatorname{sign}\left(v_{i}\right)\left(f_{i+1}-f_{i}\right)\right\} .
\end{aligned}
$$

Likewise, the new version for the second-order schemes using limiters will be defined from the following expressions:

$$
I_{i}^{n+1}=I_{i}^{n}-\lambda\left(f_{i+1 / 2}^{\mathrm{SW}+}-f_{i-1 / 2}^{\mathrm{SW}-}\right)
$$

being

$$
\begin{aligned}
& f_{i+1 / 2}^{\mathrm{SW}}=f_{i+1 / 2}^{+}+\frac{1}{2} \chi\left(r_{i+1 / 2}^{+}\right)\left[\operatorname{sign}\left(v_{i+1}\right)-\left(\lambda v_{i+1 / 2}\right)\right]\left(f_{i+1}-f_{i}\right), \\
& f_{i+1 / 2}^{\mathrm{SW}-}=f_{i+1 / 2}^{-}+\frac{1}{2} \chi\left(r_{i+1 / 2}^{-}\right)\left[\operatorname{sign}\left(v_{i}\right)-\left(\lambda v_{i+1 / 2}\right)\right]\left(f_{i+1}-f_{i}\right)
\end{aligned}
$$

and

$$
r_{i+1 / 2}^{+}=\left\{\begin{array}{ll}
\frac{I_{i}-I_{i-1}}{I_{i+1}-I_{i}} & \text { if } v_{i+1} \geqslant 0, \\
\frac{I_{i+2}-I_{i+1}}{I_{i+1}-I_{i}} & \text { if } v_{i+1}<0,
\end{array} \quad r_{i+1 / 2}^{-}= \begin{cases}\frac{I_{i}-I_{i-1}}{I_{i+1}-I_{i}} & \text { if } v_{i} \geqslant 0, \\
\frac{I_{i+2}-I_{i+1}}{I_{i+1}-I_{i}} & \text { if } v_{i}<0 .\end{cases}\right.
$$

Note that now the schemes have been defined taking into account that the flow could be coming in an element from their neighbouring elements. 


\subsubsection{A flux limiter strategy for two-dimensional problems}

Following a similar reasoning and assuming a mesh composed of triangles, we propose the following discretisation of Eq. (9) for the two-dimensional case

$$
I_{i}^{n+1}=I_{i}^{n}-\frac{\Delta t}{A(i)} \sum_{j=1}^{3} l_{i j} \underline{\hat{f}}_{i j} \cdot \underline{n}_{i j},
$$

where $A(i)$ is the area of the control volume $i, j$ represents a generic neighbouring cell (triangle), $\underline{n}_{i j}$ is the outward unit vector on the common edge of the triangles $i$ and $j$ and $l_{i j}$ is the length of that edge.

As in the one-dimensional case, the numerical fluxes defining a first-order upwind scheme in the twodimensional case could be given replacing $\underline{f}_{i j} \cdot \underline{n}_{i j}$ in Eq. (14) by

$$
\underline{\hat{f}}_{i j}^{\mathrm{UP}} \cdot \underline{n}_{i j}=\frac{1}{2}\left\{\left(\underline{f}_{i}+\underline{f}_{j}\right) \cdot \underline{n}_{i j}-\operatorname{sign}\left(\cos \left(\theta_{i j}\right)\right)\left(\underline{f}_{j}-\underline{f}_{i}\right) \cdot \underline{n}_{i j}\right\}
$$

in which $f_{k}=\underline{v}_{k} I_{k}$ for $k=i, j$, respectively, and $\theta_{i j}$ denotes the angle between $\underline{n}_{i j}$ and the velocity vector of the adjacent cell $\underline{v}_{j}$. Moreover, since

$$
\sum_{j=1}^{3} l_{i j} \underline{n}_{i j}=0
$$

for any triangle, then

$$
\sum_{j=1}^{3} l_{i j}\left(\underline{f}_{j}-\underline{f}_{i}\right) \cdot \underline{n}_{i j}=\sum_{j=1}^{3} l_{i j}\left(\underline{f}_{j}+\underline{f}_{i}\right) \cdot \underline{n}_{i j}
$$

and Eq. (14) can be rewritten as

$$
I_{i}^{n+1}=I_{i}^{n}-\frac{\Delta t}{A(i)} \sum_{j=1}^{3} l_{i j} \underline{f}_{-i j}^{-} \cdot \underline{n}_{i j},
$$

where

$$
\underline{f}_{-i j}^{-}= \begin{cases}f_{j}-\underline{f}_{i} & \text { if } \cos \left(\theta_{i j}\right)<0, \\ 0 & \text { if } \cos \left(\theta_{i j}\right) \geqslant 0 .\end{cases}
$$

We can notice in Eq. (16) that only the incoming characteristics to the control volume $i$ are considered. It is easy to verify that this new 2D scheme reduces to Eq. (13) when it is restricted to one dimension.

In order to extend the flux limiters technique for the two-dimensional case, we observe that in the present formulation and by comparison with the classical definition of the second-order Lax-Wendroff scheme in the one-dimensional case, the numerical flux function associated to the conventional second-order scheme could be expressed as

$$
\underline{\hat{f}}_{i j}^{\mathrm{LW}} \cdot \underline{n}_{i j}=\frac{1}{2}\left\{\left(\underline{f}_{i}+\underline{f}_{j}\right) \cdot \underline{n}_{i j}-\left(\frac{\Delta t}{\delta_{i j}} \underline{v}_{i j} \cdot \underline{n}_{i j}\right)\left(\underline{f}_{j}-\underline{f}_{i}\right) \cdot \underline{n}_{i j}\right\},
$$

where $\underline{v}_{i j}$ denotes an average velocity between the cells $i$ and $j$ (the arithmetic average, by example) and $\delta_{i j}$ represents the distance between the baricenters of the triangles $i$ and $j$.

Analogously, by comparing Eq. (17) with the numerical flux given by Eq. (15), the extension of the flux limiters techniques to the $2 \mathrm{D}$ case requires to replace $\hat{f}_{i j} \cdot \underline{n}_{i j}$ in Eq. (14) by

$$
\underline{\hat{f}}_{i j}^{\mathrm{SW}} \cdot \underline{n}_{i j}=\underline{\hat{f}}_{i j}^{\mathrm{UP}} \cdot \underline{n}_{i j}+\frac{1}{2} \chi\left(r_{i j}\right)\left(\operatorname{sign}\left(\cos \left(\theta_{i j}\right)\right)-\frac{\Delta t}{\delta_{i j}} \underline{v}_{i j} \cdot \underline{n}_{i j}\right)\left(\underline{f}_{j}-\underline{f}_{i}\right) \cdot \underline{n}_{i j} .
$$




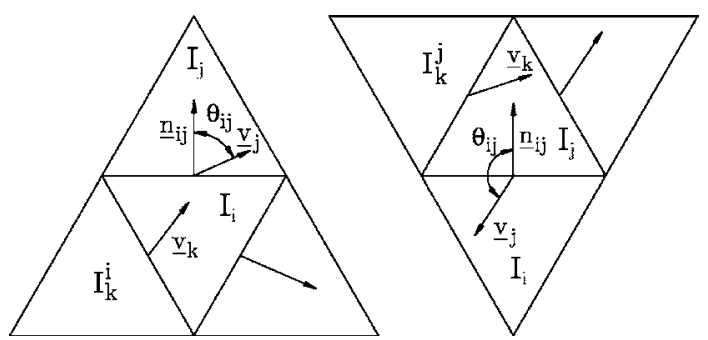

Fig. 5. Nomenclature used for the definition of $r_{i j}$.

Our strategy to define $r_{i j}$ is based on a simple heuristic 2D extension of the one proposed in the 1D case, which compare consecutive variations of the numerical solution according to the flow coming or leaving an element from their neighbouring elements

$$
r_{i j}= \begin{cases}\min _{k \neq j, \cos \left(\theta_{i k}\right)<0}\left\{\frac{I_{i}-I_{k}^{i}}{I_{j}-I_{i}}\right\} & \text { if } \cos \left(\theta_{i j}\right) \geqslant 0, \\ \min _{k \neq i, \cos \left(\theta_{j k}\right)<0}\left\{\frac{I_{k}^{j}-I_{j}}{I_{j}-I_{i}}\right\} & \text { if } \cos \left(\theta_{i j}\right)<0 .\end{cases}
$$

For a generic triangle $i$, we have denoted by $I_{k}^{i}$ (with $k \neq j$ ) the value of the volume fraction $I$ in the adjacent triangles of $i$, different to $j$. Analogously, we have denoted by $I_{k}^{j}($ with $k \neq i$ ) the value of the volume fraction $I$ in the adjacent triangles of $j$, different to $i$.

In order to detect discontinuities in the numerical solution, when the flow goes from $i$ to $j$ we compare the gradients of the solution between the triangles $i$ and $j$ with the gradients of the solution between the triangle $i$ and their adjacent triangles different to $j$ from which the flow is coming to the triangle $i$. When the flow is coming from $j$ to $i$, we compare the gradients of the solution between the triangles $i$ and $j$ with the gradients of the solution between the triangle $j$ and their adjacent triangles different to $i$ from which the flow is coming to the triangle $j$ (see Fig. 5).

\section{Numerical examples}

In order to evaluate the numerical method just proposed, we analyze both the one- and the two-dimensional cases.

\subsection{One-dimensional simulation}

Exact solutions are available in the one-dimensional case which allows a direct evaluation of the accuracy and facilitates its optimisation. A domain of $1 \mathrm{~m}$ of length is considered, where the resin is injected through the central node with an injection pressure $P_{i}=3$ bars. The preform permeability $K$ and the resin viscosity $\mu$ are assumed to be $10^{-8} \mathrm{~m}^{2}$ and $1 \mathrm{~Pa} \mathrm{~s}$, respectively. A time step of $0.1 \mathrm{~s}$ and $f(I)=I$ have been considered. At the beginning of the injection process the domain is assumed empty, excepts the two central elements that represent the injection nozzle. Assuming a constant permeability of the porous medium, the flow front position at time $t$ is given by

$$
x_{\mathrm{rf}}(t)=x_{\mathrm{IP}}+\sqrt{x_{\mathrm{r}}^{2}+2 \frac{K}{\mu} P_{i} t} ; \quad x_{\mathrm{If}}(t)=x_{\mathrm{IP}}-\sqrt{x_{1}^{2}+2 \frac{K}{\mu} P_{i} t},
$$


where $x_{\mathrm{rf}}$ and $x_{\mathrm{If}}$ represent the right and left flow front positions, $x_{\mathrm{r}}$ and $x_{1}$ their positions at $t=0$ and $x_{\mathrm{IP}}$ is the injection point location. Then, for example, the right flow front velocity and the filling time of the right part of the mold, become

$$
v_{\mathrm{f}}(t)=\frac{2 K P_{i} / \mu}{\sqrt{x_{\mathrm{r}}^{2}+2 K P_{i} t / \mu}} ; \quad t_{\text {filling }}=\frac{\left(L_{\mathrm{r}}^{2}-x_{\mathrm{r}}^{2}\right) \mu}{2 K P_{i}},
$$

where $L_{\mathrm{r}}$ is the distance from the injection point to the right mold boundary.

Fig. 6 shows the value of the volume fraction $5 \mathrm{~s}$ after the beginning of the injection, obtained with the first-order scheme, Minmod, Superbee and Monotonized limiters, using a uniform mesh of 20 elements. We can notice the very low numerical diffusion of the flow front, with only two elements located in the flow front area $(0<I<1)$ in spite of the coarse mesh used. As it can be seen in the Fig. 7, all the schemes overestimate the injected volume, being the first-order scheme the one which exhibits the highest deviation from the exact solution. The error has been defined from the following time average:

$$
\operatorname{Error}(\%)=\frac{1}{t} \int_{0}^{t}\left|\frac{\operatorname{AV}\left(t^{\prime}\right)-\operatorname{IV}\left(t^{\prime}\right)}{\operatorname{IV}\left(t^{\prime}\right)}\right| \mathrm{d} t^{\prime} \cdot 100
$$

where $\mathrm{AV}\left(t^{\prime}\right)$ denotes the VOF accumulated inside the mold at time $t^{\prime}$ (computed from the volume fraction solution at time $t^{\prime}$ ) and $\operatorname{IV}\left(t^{\prime}\right)$ denotes the exact injected volume at time $t^{\prime}$ (computed directly from the inflow boundary condition).

As expected, Fig. 8 shows that for a given time, in this case $t=25 \mathrm{~s}$, the solution accuracy increases as the elements size decreases. Moreover, we can notice that the Superbee flux limiter bring the best results.
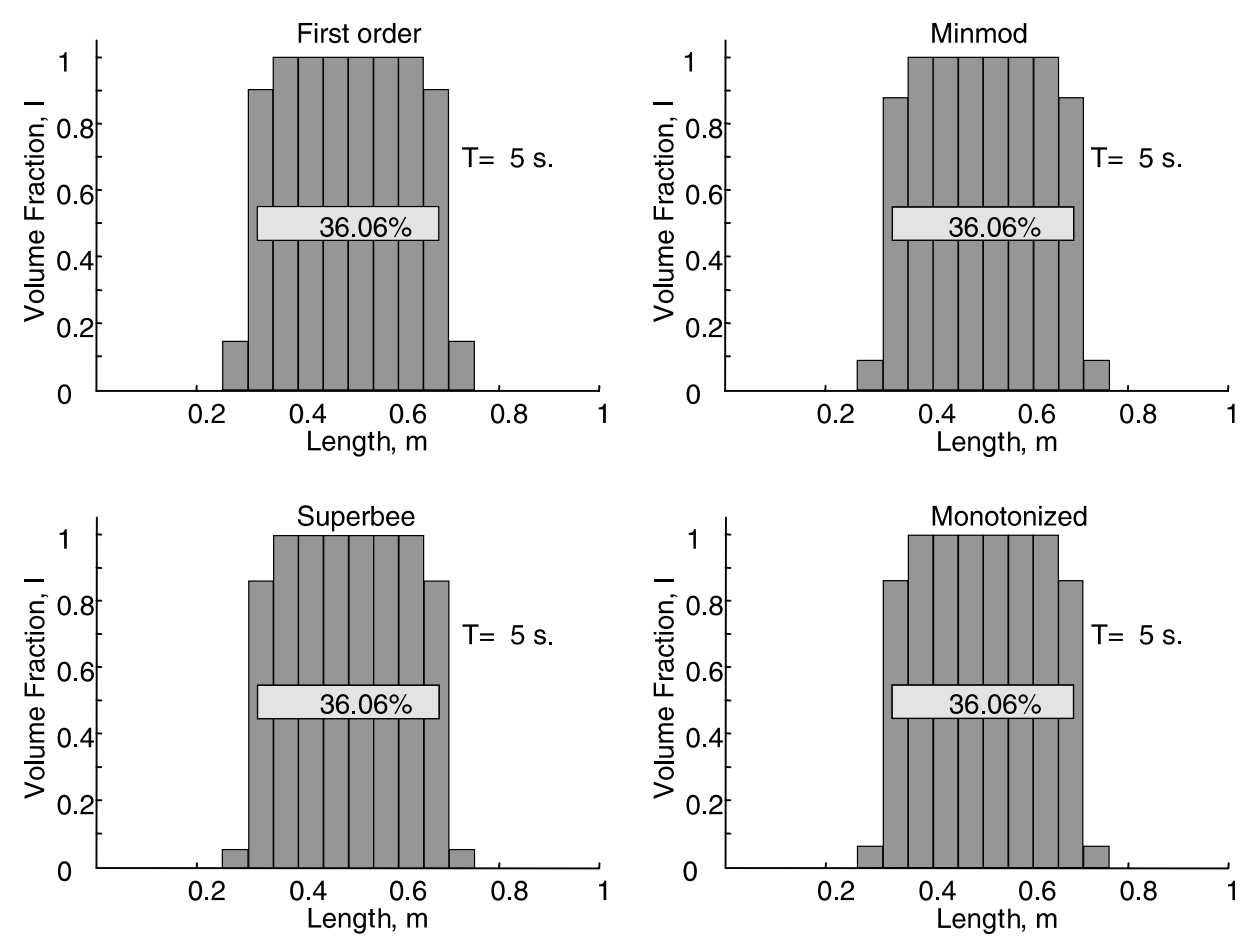

Fig. 6. Volume fraction at $t=5 \mathrm{~s}$ for different limiters. 


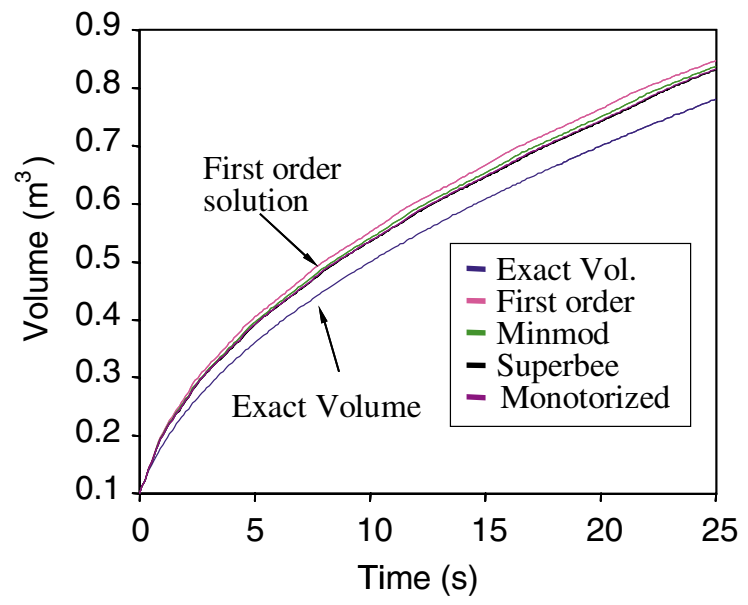

Fig. 7. Flux limiters comparison.

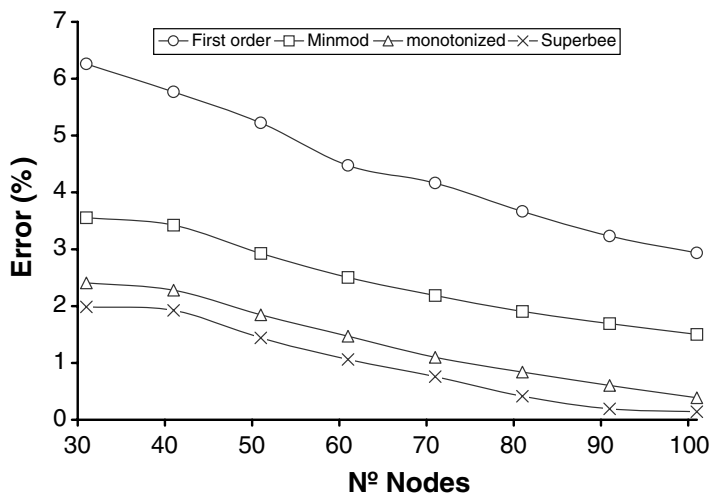

Fig. 8. Mass conservation error versus mesh size at $t=25 \mathrm{~s}$ (1D simulation).

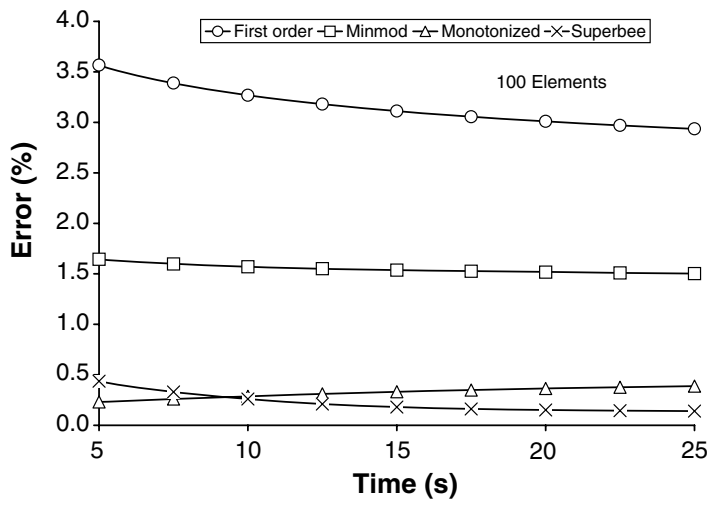

Fig. 9. Evolution of the mass conservation error during the injection process (1D simulation using a mesh of 100 elements). 
Fig. 9 depicts the evolution of the mass conservation error during the injection process, when a mesh containing 100 elements is considered.

\subsection{Two-dimensional simulations}

Now we focus on the two-dimensional evaluation of the proposed strategy. In all cases, we have used to update the volume fraction a second-order scheme with the Superbee flux limiter, because this limiter leads to the best results in the one-dimensional case.

For the two-dimensional analysis we consider a mold in which, due to its geometry and boundary conditions, the resulting flow becomes in fact unidirectional, allowing to a comparison with the exact solution.

The first mold domain considered is $1 \mathrm{~m} \times 0.4 \mathrm{~m}$. A first mesh of 80 triangles is shown in Fig. 10. The resin is injected through the left boundary with a constant flow velocity $\underline{v}_{i}=\left(10^{-3}, 0\right) \mathrm{m} / \mathrm{s}$. The preform permeability $K$ and the resin viscosity $\mu$ are $10^{-7} \mathrm{~m}^{2}$ and $0.1 \mathrm{~Pa}$, respectively.

As in the one-dimensional case, the volume fraction, $I$, is used to describe the evolution of the fluid domain. The initial fluid domain is also depicted in Fig. 10. The grey colour represents the full filled domain, whereas the white colour represents the empty domain. Since the injected flow rate is constant, we know the exact position of the flow front, which progresses linearly in time. The exact flow front position is represented in all figures with a grey horizontal bar (Fig. 10). Besides, the error between the computed accumulated volume (numerically computed) and the injected one can be calculated.

A linear weighting function $f(I)=I$ has been considered in the numerical simulations with $\alpha(I)$ adjusted to $2 \times 10^{-6}(\mathrm{Pas})^{-1}$.

Fig. 11 shows the pressure fields at times $t=200,400,600$ and $800 \mathrm{~s}$. Since the injection velocity is constant, the injection pressure increases as the flow front progresses. As it can be noticed, the pressure decreases linearly from the inflow boundary to the flow front, being zero in the empty domain, in spite of the coarse mesh considered in this simulation.

Figs. 12 and 13 represent the evolution of the mass conservation error during the injection process for two different meshes, the first one (Fig. 12) containing 80 elements and the second one (Fig. 13) with 180 elements. As expected, the error decreases as the number of element increases, being, as in the onedimensional case, the Superbee flux limiter the most accurate.

Now, we simulate a 2D mold filling processes with a radial flow. The domain used is a circular mold of $0.5 \mathrm{~m}$ of diameter, where a mesh of 512 triangles is considered. The resin is injected through a single point located at the centre of the mold. An injection pressure of 3 bars, a resin viscosity of $1 \mathrm{~Pa}$ s and an isotropic preform of permeability $K=10^{-8} \mathrm{~m}^{2}$ are considered.

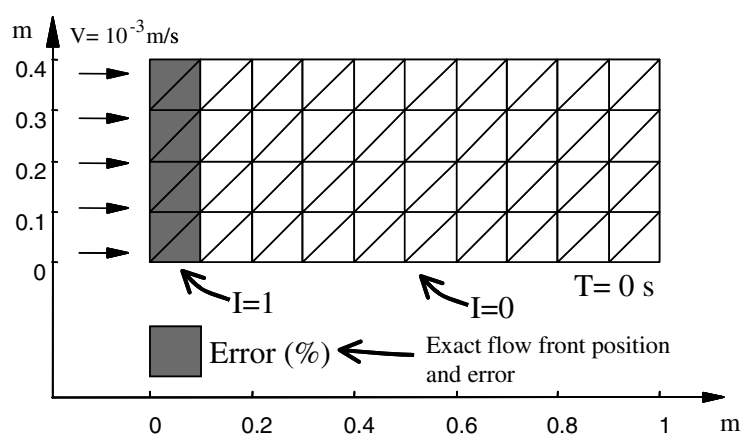

Fig. 10. Two-dimensional mold domain and Volume Fraction at $t=0$. 

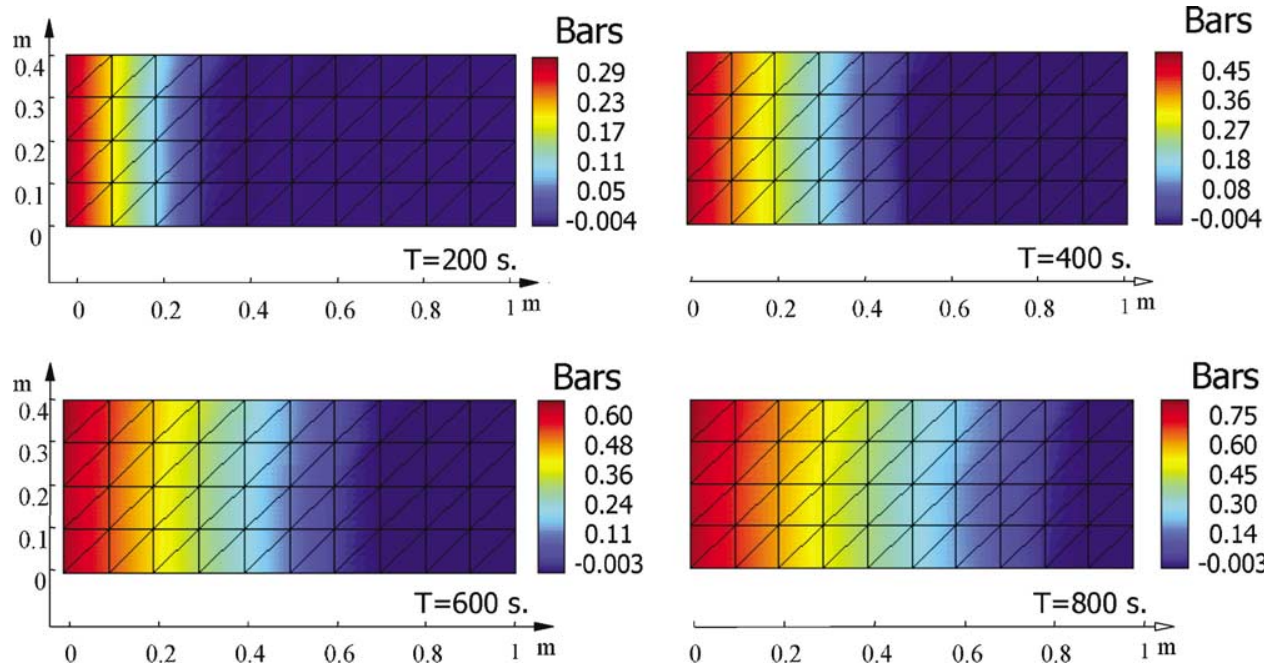

Fig. 11. Pressure field at $t=200,400,600$ and $800 \mathrm{~s}$.

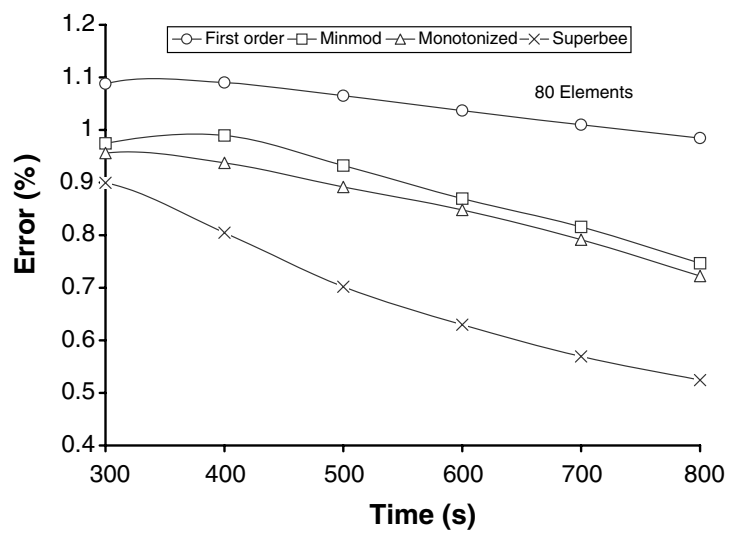

Fig. 12. 2D injection simulation: evolution of the mass conservation error using a mesh with 80 elements.

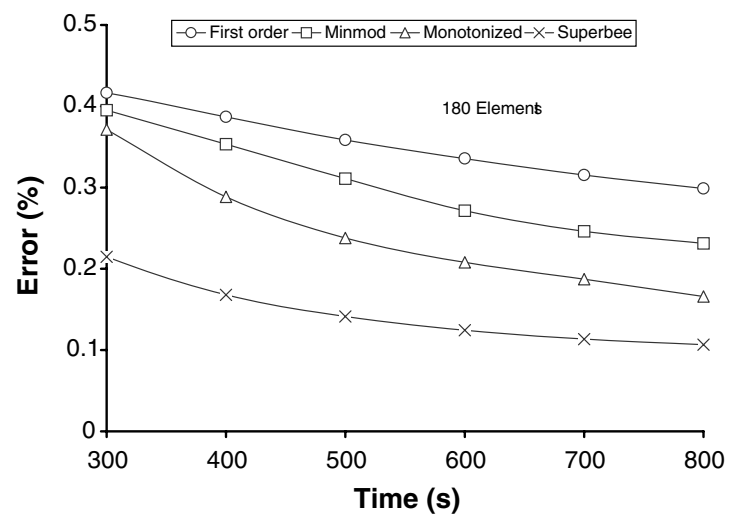

Fig. 13. 2D injection simulation: evolution of the mass conservation error using a mesh with 180 elements. 

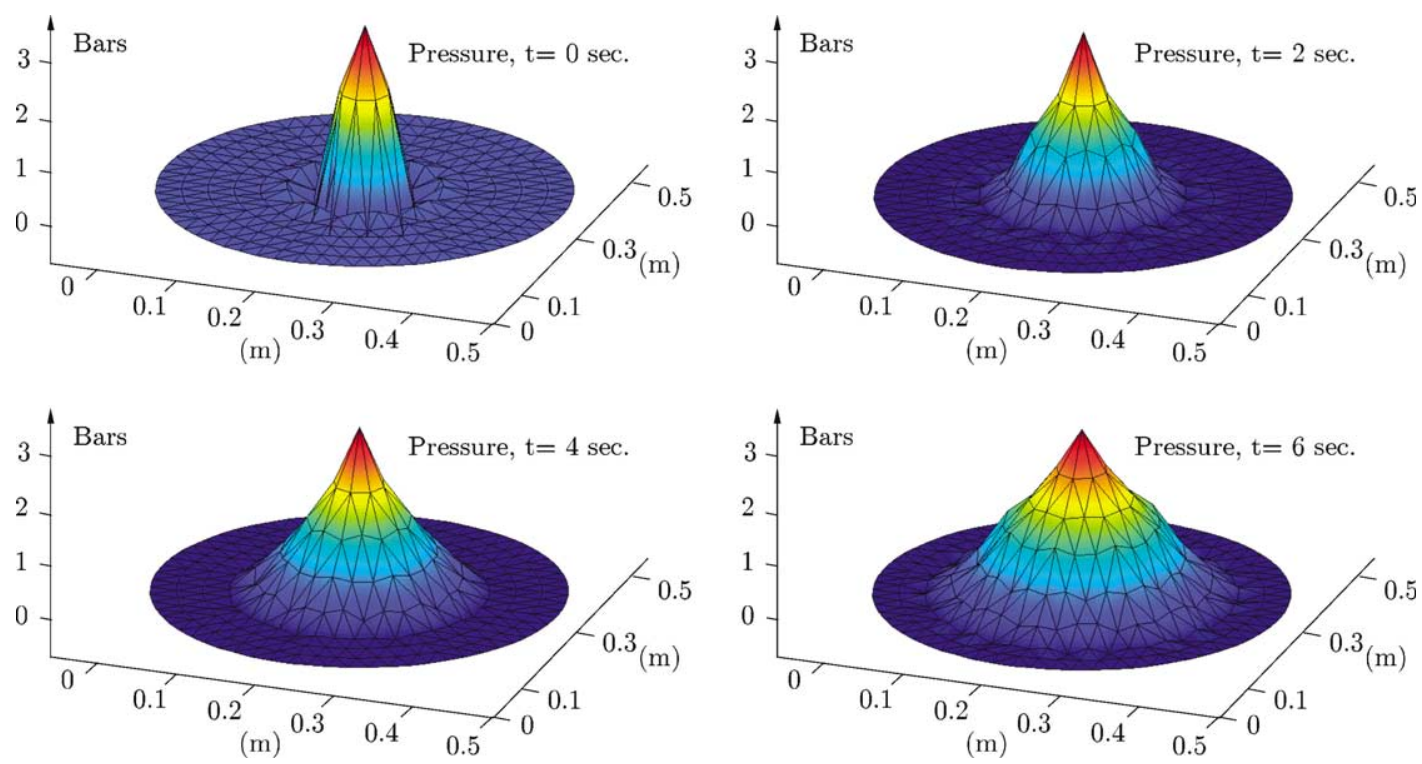

Fig. 14. Pressure field at $t=0,2,4$ and $6 \mathrm{~s}$.

Fig. 14 shows the pressure fields at $t=0,2,4$ and $6 \mathrm{~s}$. Small numerical oscillations of the pressure field appear during the first iterations, that can be removed by a local remeshing.

\section{Conclusions}

An accurate technique for porous media flow simulation has been presented. This method combines a discretisation of the fluid fraction evolution equation and the imposition of the equations of motion in a fixed mesh. The fluid fraction evolution is updated by using a second-order flux limiter scheme. The results obtained suggest that the present numerical method has a satisfactory capability of simulating the RTM process.

We have analyzed the behaviour of different flux limiters (Minmod, Superbee and Monotorized Centered) in the updating of the volume fraction. It has been pointed out that the original version of these techniques, as they appear in the literature, cannot be applied to specific cases, in particular, in multi-point injection. With this aim in view, we have proposed some improvements of those techniques that leads excellent results independently of the position of the injection point. The Superbee limiter provides the best results, improving significantly the results obtained by using the standard first-order scheme. This strategy can be also generalized to the 2D case, in the framework of a non-structured mesh of triangles.

For a complete process modelling will be necessary to introduce the energy equations as well as the resin viscosity evolution during the curing. This modelling can be easily achieved in the framework of the numerical strategy proposed in this paper.

\section{Acknowledgements}

This research work is supported by a grant from the Ministerio de Ciencia y Tecnología (MCYT) and another one from the Fondo Europeo de Desarrollo Regional (FEDER), proyect DPI2001-2792. 


\section{References}

[1] S.G. Advani, Flow and Rheology in Polymer Composites Manufacturing, Elsevier, 1994.

[2] J.P. Boris, D.L. Book, Flux-corrected transport I. SHASTA. A fluid transport algorithm that works, J. Comput. Phys. 11 (1973) $38-69$.

[3] D.L. Book, J.P. Boris, K. Hain, Flux-corrected transport II: generalizations of the method, J. Comput. Phys. 18 (1975) $248-283$.

[4] J.A. García, F. Chinesta, Ll. Gascón, I. Ordeig, A pseudo-domain technique for simulating flows through porous media, in: 36e Colloque Annuel du Groupe Français de Rhéologie, 2001, pp. 243-249.

[5] J.A. García, Gestión del Frente de Avance en la Modelización Numérica del Conformado por Transferencia de Resina (RTM), Ph.D. Thesis, Departamento de Ingeniería Mecánica y de los Materiales, Universidad Politécnica de Valencia, 2000.

[6] Ll. Gascón, J.M. Corberán, TVD schemes for non-homogeneous conservation laws, J. Comput. Phys. 172 (2001) $261-297$.

[7] A. Harten, High resolution schemes for hyperbolic conservation laws, J. Comput. Phys. 49 (1983) 357-393.

[8] C. Hirsch, Numerical Computation of Internal and External Flows, Computational Methods for Inviscid and Viscous Flows, vol. 2, Wiley, Chichester, 1990.

[9] C.W. Hirt, B.D. Nichols, Volume of fluid (VOF) method for the dynamics of free boundaries, J. Comput. Phys. 39 (1981) $201-225$.

[10] R. Leveque, Numerical Methods for Conservation Laws, Birkhäuser, Basel/Boston/Berlin, 1990.

[11] S. Li, R. Gauvin, Numerical analysis of the resin flow in resin transfer molding, J. Reinforced Plastics Compos. 10 (3) (1990) 314.

[12] P.K. Sweby, High resolution schemes using flux limiters for hyperbolic conservation laws, SIAM J. Numer. Anal. (1984) 9951011.

[13] T.E. Tezduyar, M. Behr, S. Mittal, A.A. Johnson, Computation of unsteady incompressible flows with the stabilized finite element methods, in: Space-Time Formulations, Iterative Strategies and Massively Parallel Implementations, New Methods in Transient Analysis, AMD-Vol 143, ASME, 1992, pp. 7-24.

[14] T.E. Tezduyar, Finite element interface-tracking and interface capturing techniques for flows with moving boundaries and interfaces, in: Proceedings of 2001 ASME International Mechanical Engineering Congress and Exposition, New York, 2001.

[15] K. Stein, T.E. Tezduyar, Advanced mesh update techniques for problems involving large displacements, Fifth World Congress on Computational Mechanics, Vienna, Austria, 2002.

[16] T.E. Tezduyar, Finite element methods for flow problems with moving boundaries and interfaces, Arch. Comput. Meth. Engrg. 8 (2001) 83-130.

[17] E.F. Toro, Riemann Solvers and Numerical Methods for Fluid Dynamics, Springer-Verlag, New York, 1997.

[18] F. Trochu, R. Gauvin, D.M. Gao, Numerical analysis of the resin transfer moulding process by the finite element method, Adv. Polym. Technol. 12 (4) (1993) 329-342.

[19] B. Van Leer, Towards the ultimate conservative difference scheme II. Monotonicity and conservation combined in a second order scheme, J. Comput. Phys. 14 (1974) 361-370.

[20] B. Van Leer, Towards the ultimate conservative difference scheme V. A second-order sequel to Godunov's method, J. Comput. Phys. 32 (1979) 101-136. 